This item was submitted to Loughborough's Research Repository by the author.

Items in Figshare are protected by copyright, with all rights reserved, unless otherwise indicated.

\title{
Adaptive partial update channel shortening in impulsive noise environments
}

PLEASE CITE THE PUBLISHED VERSION

PUBLISHER

(C) IEEE

VERSION

VoR (Version of Record)

LICENCE

CC BY-NC-ND 4.0

REPOSITORY RECORD

Grira, Mahmud, and Jonathon Chambers. 2019. "Adaptive Partial Update Channel Shortening in Impulsive Noise Environments". figshare. https://hdl.handle.net/2134/5634. 
This item was submitted to Loughborough's Institutional Repository (https://dspace.lboro.ac.uk/) by the author and is made available under the following Creative Commons Licence conditions.

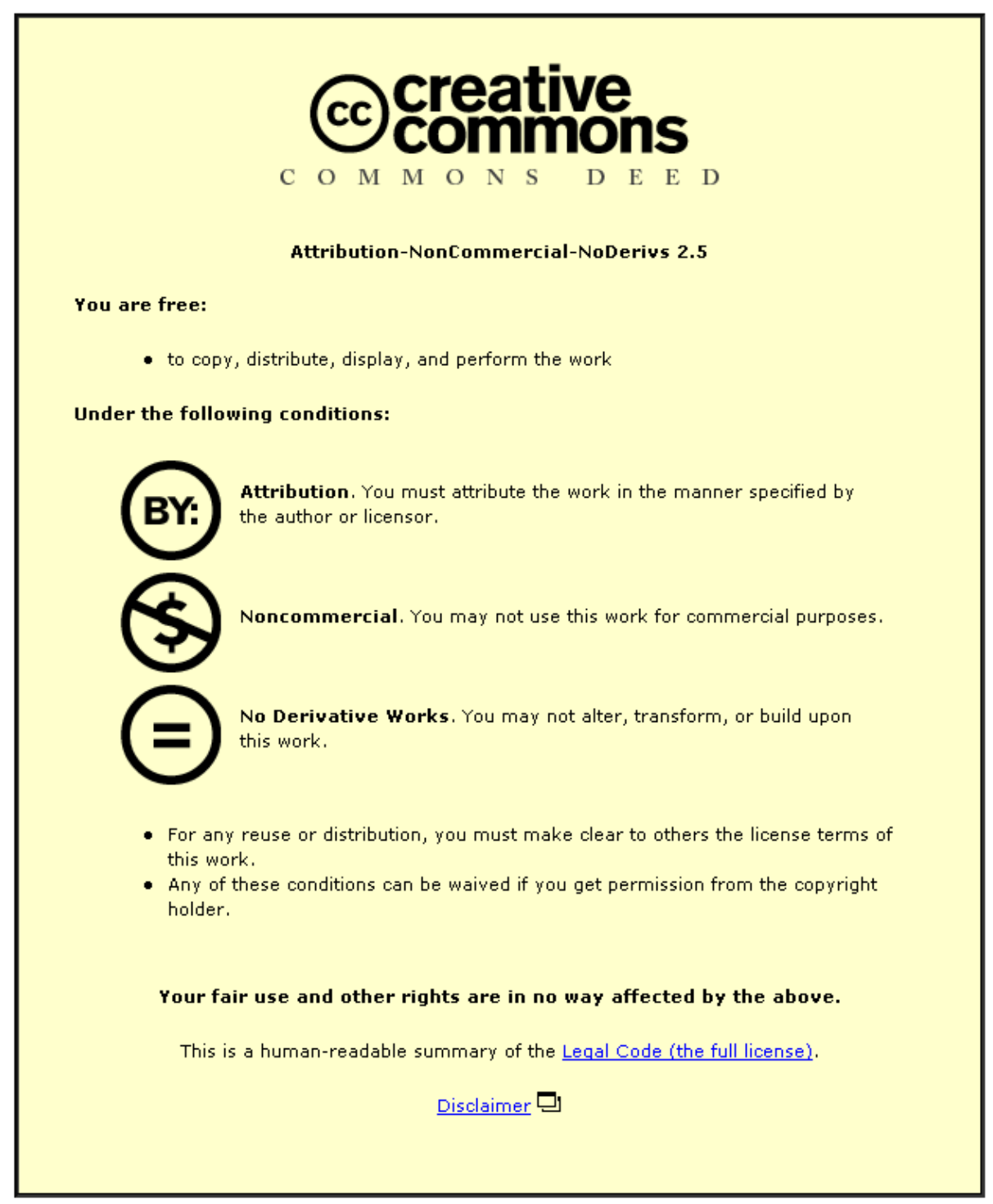

For the full text of this licence, please go to: http://creativecommons.org/licenses/by-nc-nd/2.5/ 


\title{
ADAPTIVE PARTIAL UPDATE CHANNEL SHORTENING IN IMPULSIVE NOISE ENVIRONMENTS
}

\author{
Mahmud Grira, Jonathon A. Chambers \\ Center of Digital Signal Processing \\ Cardiff School of Engineering Queen's Buildings \\ PO Box 925, Cardiff Cf24 0YF, Wales,UK \\ griram, chambersj@cf.ac.uk
}

\begin{abstract}
Partial updating is an effective method for reducing computational complexity in adaptive filter implementations. In this paper adaptive partial update channel shortening algorithms in impulsive noise environments are proposed. These algorithms are based on updating a portion of the coefficients at each time sample instead of the entire set of coefficients. These algorithms have low computational complexity whilst retaining essentially identical performance to the sum-absolute autocorrelation minimization (SAAM) algorithm due to Nawaz and chambers. Simulation studies show the ability of the deterministic partial update SAAM (DPUSAAM) algorithm and the Random Partial Update SAAM (RPUSAAM)algorithm to achieve channel shortening and hence an acceptable level of bitrate within a multicarrier system.
\end{abstract}

\section{INTRODUCTION}

In multicarrier modulation systems (MCM), such as asymmetrical digital subscriber line (ADSL) transceivers, each symbol consists of samples to be transmitted to the receiver plus a cyclic prefix (CP) of length $\mathrm{v}$ [1]. The $\mathrm{CP}$ is the last $\mathrm{v}$ samples of the original $\mathrm{N}$ samples to be transmitted. The CP is inserted between blocks to combat intersymbol interference (ISI) and inter-channel interference (ICI). The length of the $\mathrm{CP}$ should at least be equal to the order of the channel impulse response. At the receiver the $\mathrm{CP}$ is removed, and the remaining $\mathrm{N}$ samples are then processed by the receiver. To combat ISI, the impulse response of the channel must be of length $v+1$ or shorter than a CP of length v. Since the efficiency of the transceiver is reduced by the introduction of the $\mathrm{CP}$ it is therefore desirable either to make $\mathrm{v}$ as small as possible or to choose a large $\mathrm{N}$. Selecting large $\mathrm{N}$ will increase the computational complexity, system delay, and memory requirements of the transceiver. Also the length of the channel's impulse response varies from channel to channel, so to achieve reasonable efficiency a large $\mathrm{v}$ and large $\mathrm{N}$ have to be chosen. To overcome these problems a short time-domain equalizer (TEQ), usually an FIR filter, is typically placed in the receiver. The purpose of this filter is to shorten the impulse response of the effective channel. The impulse response of the effective channel needs to be shorter than the length of the CP. The length of the shortened impulse response filter and $\mathrm{CP}$ are usually fixed a priori and not changed from channel to channel. A low complexity blind adaptive algorithm to design a TEQ, called sum-squared auto-correlation minimization (SAM) was proposed in [2] which achieves channel shortening by minimizing the sum-squared autocorrelation terms of the effective channel impulse response outside a window of a desired length. The drawback with SAM is that it has a significant computational complexity,and it is not robust to impulse noise, whereas the SAAM algorithm is more robust to impulse noise by minimizing the sum of the absolute values (instead of sum of squared values) outside a window of desired length [3]. But SAAM did not reduce the computational complexity of SAM. In this paper, we propose new partial update algorithms in which the filter coefficients that are updated every iteration are selected in a deterministic way and also at random, with much reduced computational complexity. Simulation studies confirm that little performance degradation results from the proposed computationally efficient algorithms.

\section{SYSTEM MODEL}

The system model is shown in Figure (1). The input signal $x(n)$ typically drawn from a finite constellation is the source sequence to be transmitted through a linear finite-impulse-response (FIR) channel $\mathbf{h}$ of length $\left(L_{h}+1\right)$ taps. $r(n)$ is the received signal, which will be filtered through an $\left(L_{w}+1\right)$-tap TEQ with an impulse response vector $\mathbf{w}$ to obtain the output sequence $y(n)$. We denote $\mathbf{c}=\mathbf{h} * \mathbf{w}$ as the shortened or effective channel assuming $\mathbf{w}$ is in steady-state. We also assume that $2 L_{c}<N$ holds. The signal $v(n)$ is a zero-mean, i.i.d., noise sequence uncorrelated with the source sequence which has variance $\sigma_{v}^{2}$. The received sequence $r(n)$ is

$$
r(n)=\sum_{k=0}^{L_{h}} h(k) x(n-k)+v(n)
$$

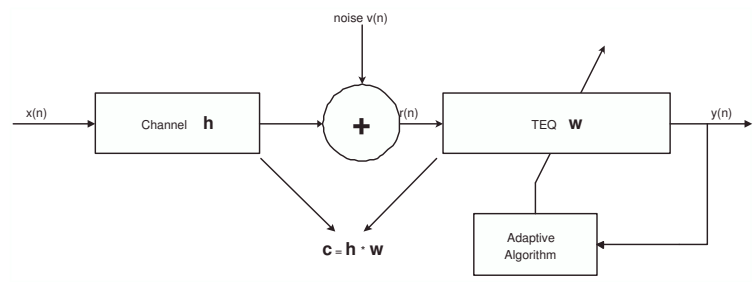

Fig. 1. System model for blind adaptive channel shortening.

and the output of the TEQ $y(n)$ is given by

$$
y(n)=\sum_{k=0}^{L_{w}} w(k) r(n-k)=\mathbf{w}^{T} \mathbf{r}_{n}
$$




$$
\begin{gathered}
\mathbf{w}^{k+1}=\mathbf{w}^{k}-\mu \times \sum_{l=v+1}^{L_{c}}\left[\operatorname{sign}\left\{\sum_{n=k N}^{(k+1) N-1} \frac{y(n) y(n-l)}{N}\right\} \times\left\{\sum_{n=k N}^{(k+1) N-1}\left(\frac{y(n) \mathbf{r}(n-l)+y(n-l) \mathbf{r}(n)}{N}\right)\right\}\right] \\
\mathbf{w}^{k+1}=\mathbf{w}^{k}-\mu \times M_{k} \times \sum_{l=v+1}^{L_{c}}\left[\operatorname{sign}\left\{\sum_{n=k N}^{(k+1) N-1} \frac{y(n) y(n-l)}{N}\right\} \times\left\{\sum_{n=k N}^{(k+1) N-1}\left(\frac{y(n) \mathbf{r}(n-l)+y(n-l) \mathbf{r}(n)}{N}\right)\right\}\right] \\
\mathbf{w}^{k+1}=\mathbf{w}^{k}-\mu \times R_{k} \times \sum_{l=v+1}^{L_{c}}\left[\operatorname{sign}\left\{\sum_{n=k N}^{(k+1) N-1} \frac{y(n) y(n-l)}{N}\right\} \times\left\{\sum_{n=k N}^{(k+1) N-1}\left(\frac{y(n) \mathbf{r}(n-l)+y(n-l) \mathbf{r}(n)}{N}\right)\right\}\right]
\end{gathered}
$$

where $\mathbf{r}_{n}=\left[r(n) \quad r(n-1) \cdots r\left(n-L_{w}\right)\right]^{T}$ and $\mathbf{w}$ is the impulse response vector of the TEQ $\mathbf{w}=\left[\begin{array}{lll}w_{0} & w_{1} & w_{2} \cdots w_{L_{w}}\end{array}\right]^{T}$, and $(.)^{T}$ denotes vector transpose.

\section{THE NOTION OF THE SAAM ALGORITHM}

The idea of SAAM is based on minimizing the (non-negative) autocorrelation of a channel for lags $v+1$. The cost function, $J_{v+1}$ based on the sum of absolute values of the autocorrelation of the effective channel is suggested [3]. The reasons for taking absolute values have been explained in [3]. This is in contrast to the cost function of [2] based on the sum of squared autocorrelation values for the same lags. The autocorrelation sequence of the effective channel, $\mathbf{c}$ is given by

$$
R_{c c}(l)=\sum_{k=0}^{L_{c}} c(k) c(k-l)
$$

when the effective channel $\mathbf{c}$ has zero taps outside a window of size $(v+1)$, and for a shortened channel, it must satisfy,

$$
R_{c c}(l)=0, \forall|l|>v
$$

Then the cost function $J_{v+1}$ in SAAM is defined based upon minimizing the sum-absolute autocorrelation terms, i.e.,

$$
J_{v+1}=\sum_{l=v+1}^{L_{c}}\left|R_{c c}(l)\right|
$$

The update equation for SAAM can be written as in (1). The algorithm approaches the maximum shortening SNR (SSNR) solution of [1] under additive white Gaussian Noise (AWGN) condition, and is also robust to non-Gaussian impulsive noise environments.

\section{DPUSAAM}

As in any partial update algorithm, the aim of partial updating is to update a portion of the coefficients instead of the entire set of coefficients. Our proposal here is to apply the partial update method to the channel shortening algorithm (Blind, Adaptive Channel Shortening By Sum-absolute Auto-Correlation Minimization SAAM) algorithm and achieve the same performance whilst reducing the computational complexity, the proposed algorithm is called the Deterministic Partial Update SAAM algorithm (DPUSAAM). In this algorithm the coefficients in the middle (in our simulation case eight will be the middle) are updated $N_{B}-1$ times, that is achieved by introducing a vector which contains ones in the middle and zeros remainder, then at the $N_{B^{t h}}$ time the outside ones are updated. The new vectors called "mask $k_{1}$ " and "mask $k_{2}$ " are created as Mask $=[0000111111110000]$ Mask $_{2}=[1111000000001111]$. We define matrices $M_{k}=\operatorname{diag}\left(\operatorname{Mask}_{k}\right)$, where $k=1,2$. The deterministic partial-update SAAM (DPUSAAM) algorithm can therefore be written as in (2). In this work $N_{B}=5$, so that for the $N_{B}-1$ times $M_{k}=M_{1}$ otherwise $M_{k}=M_{2}$. The proposed algorithm essentially achieves the same performance as the SAAM algorithm in terms of higher bit rates and shortening the channel as will be shown in the simulation results, the advantage of the proposed algorithm is that it essentially achieves the same performance whilst updating only half of the coefficients at each iteration which implies less computational complexity.

\section{RPUSAAM}

Our proposal here is to improve the deterministic partial update scheme to exploit improved convergence of random selection [4], and achieve performance close to SAAM. The set of indices of the coefficients of the adaptive filter is given by $\left\{1,2, \ldots L_{w}+1\right\}$. This set is split into $P$ different disjoint subsets denoted $S_{i}, i=1, \ldots . . P$. Then, at each iteration one of these subsets is selected at random with probability $1 / P$, and only those coefficients within the adaptive filter having indices from that subset are updated. The update equation can be written as in (3), where $R_{k}$ is a diagonal with unity elements on the principle diagonal corresponding to the chosen subset $S_{i}$ and zeros elsewhere; and $\underline{w}(0)$ is initialized as for SAAM.

\section{ALPHA-STABLE NOISE MODULE}

The impulsive noise can be represented by a model called the $\alpha$ stable distribution [5]. This distribution also shares several desirable properties with the Gaussian model, such as the stability property and generalized form of the Central Limit Theorem [6]. $\alpha$-stable distributions also include the Gaussian density as a special case and have other useful properties. A random variable $x$ is said to have a stable distribution, denoted by $x \sim S_{\alpha}(\gamma, \beta, a)$, if the Fourier Transform of its pdf, also called its characteristic function, has the following form [5].

$$
\varphi(t)=\exp \left\{j a t-\gamma|t|^{\alpha}[1+j \beta \operatorname{sign}(t) w(t, \alpha)]\right\}
$$

where

$$
\begin{gathered}
w(t, \alpha)=\left\{\begin{array}{cc}
\tan \frac{\alpha \pi}{2}, & \text { if } \alpha \neq 1 \\
\frac{2}{\pi} \log |t|, & \text { if } \alpha=1
\end{array}\right. \\
\operatorname{sign}(t)=\left\{\begin{array}{cc}
1, & \text { if } t>0 \\
0, & \text { if } t=0 \\
-1, & \text { if } t<0
\end{array}\right.
\end{gathered}
$$

Thus the four parameters $-\infty<a<\infty, \gamma>0,0<\alpha \leq$ $2,-1 \leq \beta \leq 1$ describing the stable distribution are [5]

- $\alpha$ which is called the characteristic exponent and determines the thickness of the tails of the distribution. Smaller values of $\alpha$ correspond to heavier tailed distributions and vice versa. An $\alpha=2$ corresponds to a Gaussian distribution. Another special case is the Cauchy distribution when $\alpha=1$ and $\beta=$ 0 .

- $\gamma$ which is a scale parameter called the dispersion. It is similar to variance of a Gaussian distribution and equals half the variance in the Gaussian case. 
- $\beta$ which is a symmetry parameter. When $\beta=0$, it corresponds to a symmetric distribution. The resulting distribution is called a Symmetric $\alpha$-Stable $(\mathrm{S} \alpha \mathrm{S})$ distribution.

- $a$ which is the location parameter. It is the mean if $1<\alpha \leq 2$ and the median if $0<\alpha<1$.

\section{SIMULATION RESULTS}

The Matlab code at [7] was extended to simulate DPUSAAM, and RPUSAAM in impulsive noise environments ( $\alpha$-stable noise). The cycle prefix was 32 , the FFT size $N_{f f t}=512$, the TEQ had 16 taps and the channel was the test ADSL channel CSA loop1 available at [8]. The geometric-SNR (G-SNR) definition is used instead of the standard SNR definition, due to infinite variance of $\mathrm{S} \alpha \mathrm{S}$ distribution [9], a total of 75 OFDM symbols was used. The step size used was 0.0007 . The dispersion of the noise for a given value of $\alpha$ is changed and the quasi achievable bit rate (quasi because the same bit rate calculation, to a first approximation, on the basis that the impulses occur infrequently, is used. These results are for an impulsive contribution of $1 \%$ only) is calculated. In figures (2) and (3) the impulsive response of the original and the shortened channel with different values of $\alpha(\alpha=1.95,1.9$, these values are between the less impulsive case of Gaussian noise when $\alpha=2$ and the more impulsive Cauchy case when $\alpha=1$ [5], these values were chosen to show the robustness of the proposed algorithms) show that all algorithms are confirmed to be effective. Quasi achievable bits per second as a function of the averaging block number are plotted at $\alpha=1.95$ and 1.9 and is shown in figures (4) and (5), it can be seen that the proposed algorithms are robust to the impulsive noise as SAAM algorithm with half of the coefficients being updated. Careful inspection of figures (4) and (5) reveals the improved final performance of the random update selection scheme.

\section{CONCLUSION}

New partial update blind channel shortening algorithms in impulsive noise environments have been proposed. The proposed algorithms PUSAAM and RPUSAAM have proven to be robust in impulsive noise environments. The proposed algorithms essentially achieve the same result in terms of reducing the effective channel length as SAAM with half the complexity,

\section{REFERENCES}

[1] P. J. W. Melsa, R. C. Younce, and C. E. Rohrs, "Impulse response shortening for discrete multitone transceivers," IEEE Trans. Commun., vol. 44, pp. 1662-1672, December 1996.

[2] J. Balakrishnan, R. K. Martin, and C. R. Johnson, "Blind, adaptive channel shortening by sum-squared auto-correlation minimization (SAM)," IEEE Trans. Signal Processing, vol. 51, pp. 3086-3090, December 2003.

[3] R. Nawaz and J.A. Chambers, "Robust blind channel shortening in impulsive noise environments," 12th European Signal Processing Conf. (Eusipco), Vienna, Austria,, 2004.

[4] M. Godavarti and A. O. Hero, "Partial update LMS algorithms," IEEE Trans. on Signal Processing, vol. 53, pp. 2382-2399, July 2005.
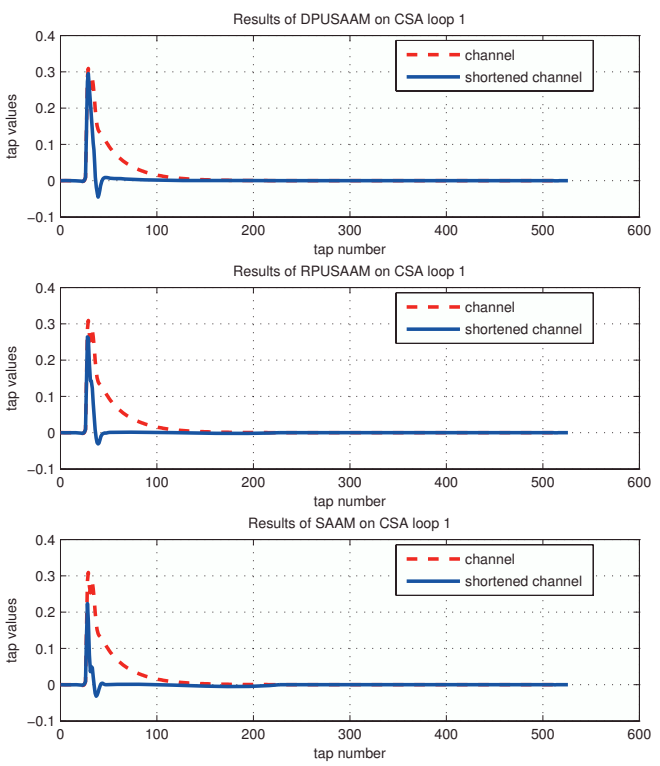

Fig. 2. Original and the shortened channel where alpha=1.95.

[5] P. G. Georgiou, P. Tsakalides, and C. Kyriakakis, "Alpha-stable modeling of noise and robust time-delay estimation in the presence of impulsive noise," IEEE Trans. on Multimedia, vol. 1, pp. 291-301, September 1999.

[6] T. C. Chuah, "Robust Techniques For Multiuser CDMA Communications In Non-Gaussian Noise Environments," Ph. D. Thesis, University of Newcastle upon Tyne, UK, 2002.

[7] R. K. Martin, "Matlab Code for Papers by R. K. Martin.[Online]. Available: http://bard.ece.cornell.edu/matlab/martin/index.html," .

[8] G. Arslan, M. Ding, B. Lu, Z. Shen, and B. L. Evans, "TEQ Design Toolbox. Univ. Texas, Austin, TX. [Online.] Available: http://www.ece.utexas.edu/ bevans/projects/adsl/ dmtteq/dmtteq.html," .

[9] J. G. Gonzalez, D. W. Griffith, and G. R. Arce, "Zero-order statistics: A signal processing framework for very impulsive environments," in Proc. IEEE Signal Proc. Workshop on Higher Order Statistics, Banff, Alberta, Canada, 1997. 

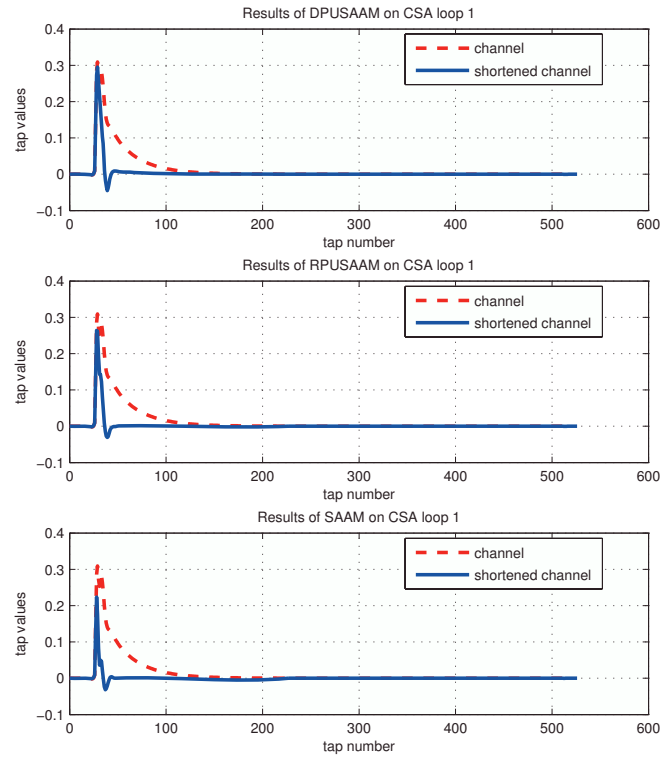

Fig. 3. Original and the shortened channel where alpha=1.9.
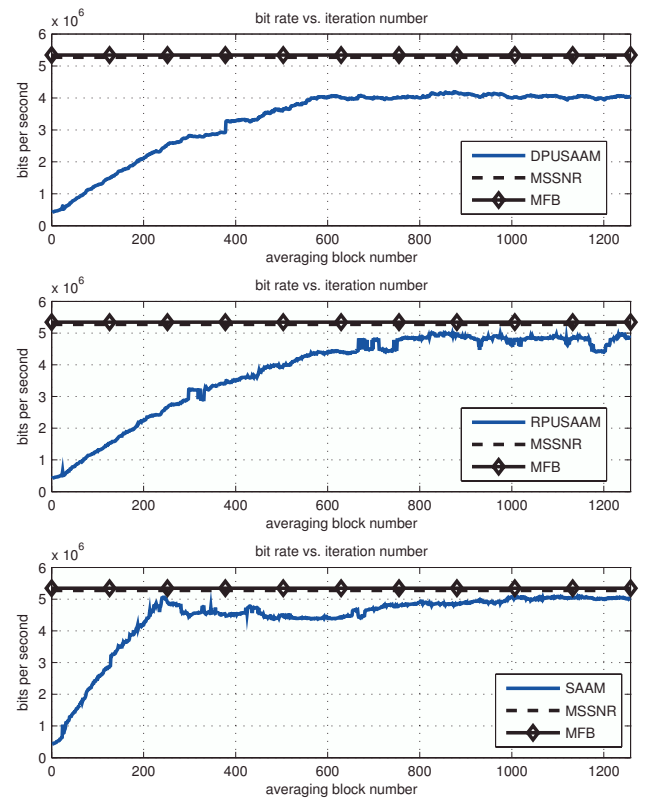

Fig. 4. Quasi-achievable bit rate versus averaging block number where alpha $=1.95$.
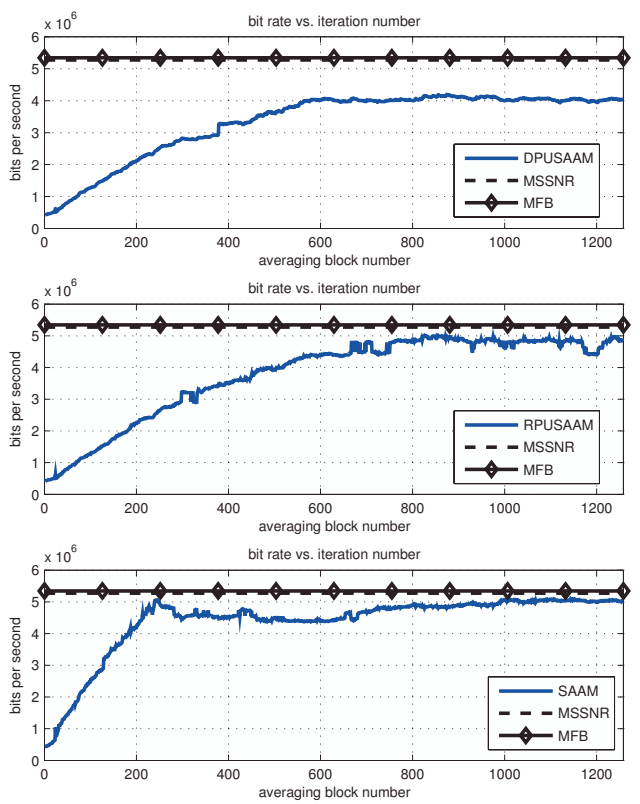

Fig. 5. Quasi-achievable bit rate versus averaging block number where alpha $=1.9$. 